BMJ Open Diabetes Research \& Care

\section{Presentation, pathology and prognosis of renal disease in type 2 diabetes}

Jasmine Tan, ${ }^{1,2}$ L Jonathan Zwi, ${ }^{3}$ John F Collins, ${ }^{4}$ Mark R Marshall, ${ }^{5}$ Tim Cundy ${ }^{1,6}$
To cite: Tan J, Zwi LJ, Collins JF, et al. Presentation, pathology and prognosis of renal disease in type 2 diabetes. BMJ Open Diab Res Care 2017;5:e000412. doi:10.1136/ bmjdrc-2017-000412

- Additional material is published online only. To view please visit the journal online (http://dx.doi.org/10.1136/ bmjdrc-2017-000412).

Received 4 March 2017 Revised 7 July 2017 Accepted 11 July 2017

\section{(a) CrossMark}

${ }^{1}$ Auckland Diabetes Centre Green Lane Clinical Centre, Auckland, New Zealand ${ }^{2}$ Department of Renal Medicine, North Shore Hospital, Auckland, New Zealand

${ }^{3}$ Department of Anatomical Pathology, Auckland City Hospital, Auckland, New Zealand

${ }^{4}$ Department of Renal Medicine, Auckland City Hospital, Auckland, New Zealand

${ }^{5}$ Department of Renal Medicine, Middlemore Hospital, Auckland, New Zealand

${ }^{6}$ Department of Medicine, Faculty of Medical \& Health Sciences, University of Auckland, Auckland, New Zealand

Correspondence to Dr Jasmine Tan; jasmine.tan@waitematadhb. govt.nz

\section{ABSTRACT}

Objective Non-diabetic renal disease (NDRD) is common in patients with type 2 diabetes (T2D), but the relationship between its presentation and prognosis is unknown.

Research design and methods In a retrospective cohort study, we compared renal and patient survival among 263 patients with T2D who had native renal biopsies between 2002 and 2008 from three Auckland hospitals in New Zealand. The presence of diabetic nephropathy (DN), NDRD or mixed (DN and NDRD) was determined from biopsy. We examined clinical associations according to NDRD etiologies and mode of presentation-acute (defined by acute kidney injury (AKI)) or non-acute. Patients were followed until end-stage renal disease, death or December 2015. Survival was compared using Log-rank test. Results 94 (36\%) patients had DN, 72 (27\%) had NDRD, and $97(37 \%)$ had mixed pathologies. Obesityrelated focal segmental glomerulosclerosis was the most common NDRD (46\%) in patients with non-acute presentations, whereas interstitial nephritis or immunecomplex glomerulonephritides were the most prevalent in those with acute presentations (60\%). DN was commonly associated with AKI $(p<0.001)$. The prevalence of DN increased with diabetes duration $(p<0.001)$, but NDRD was still found in $55 \%$ of subjects with $\geq 14$ years T2D. NDRD was strongly associated with the absence of retinopathy $(p<0.001)$. Renal survival was best in the NDRD group $(p<0.001)$. Among those with DN, renal prognosis was worse in those with more advanced DN lesions and those with an acute presentation $(p<0.001)$. The proportion of all-cause mortality was similar in all three groups, but overall survival was poorest in the DN group $(p=0.025)$. Conclusions Renal disease in patients with T2D is heterogeneous. The renal prognosis differs markedly according to histopathological diagnosis and mode of presentation.

\section{INTRODUCTION}

The prevalence of chronic kidney disease (CKD) in people with type 2 diabetes (T2D) is three times higher than in the non-diabetic population, ${ }^{1}$ and in many parts of the world, the majority of people developing end-stage renal failure (ESRD) have T2D.

The natural history of diabetic nephropathy $(\mathrm{DN})$ in patients with type 1 diabetes is well described, but renal disease in T2D appears to be more complex and heterogeneous. ${ }^{2}$ This suggests that pathologies other than, or coexisting with $\mathrm{DN}$, are important.

\section{Significance of this study}

What is already known about this subject?

- Non-diabetic renal disease is known to be prevalent in patients with type 2 diabetes.

- A number of studies have suggested that nondiabetic renal disease may have a better prognosis than classical diabetic nephropathy, but these studies have not considered whether biopsy was undertaken because of an acute deterioration in renal function or because of clinical features atypical for diabetic nephropathy.

\section{What are the new findings?}

- The new findings from our study are that the types of non-diabetic kidney disease and the renal prognosis vary with the mode of presentation.

- Patients with acute kidney injury, particularly those in whom it is superimposed on diabetic nephropathy, have a poorer renal prognosis. In those with non-acute presentations, in whom prognosis is better, focal segmental glomerulosclerosis (which was strongly associated with obesity) was the commonest non-diabetic renal disease.

How might these results change the focus of research or clinical practice?

- Provide an overview of the renal prognosis of the heterogeneous nephropathies in patients with type 2 diabetes.

- Suggest that timely renal biopsy and nephrology referral can lead to change in treatment.

- Provide a platform for further research into the natural history of nephropathy in type 2 diabetes and to identify modifiable risk factors associated with acute kidney injury in those with diabetic nephropathy.

A pooled meta-analysis of renal biopsy findings in studies predominantly consisting of patients with T2D has described a high prevalence of non-diabetic pathologies (estimates range $3 \%-83 \%$ ) with a wide variety of renal pathologies. ${ }^{3}$ However, these are not true prevalence estimates as renal biopsy is not routinely undertaken in patients with T2D and renal disease.

Patients with T2D selected for renal biopsy are typically those with a presentation that is out of keeping with 'classical' DN. These 
indications can be grouped broadly into either acute presentations, with a rapid loss of renal function over a short period (acute kidney injury (AKI)) or non-acute presentations in which there are atypical clinical features.

Proteinuric renal disease and diabetes are both associated with heightened risk of AKI and the occurrence of AKI increases the risk for progressive CKD and ESRD. ${ }^{45}$ Thus, it is surprising that a number of small studies have suggested that patients with NDRD might have a better prognosis than those with classical DN. ${ }^{6-9}$ These studies have not, however, distinguished the types of presentation and pathologies that are associated with a better prognosis. To better understand the nature and significance of NDRD, and its interaction with DN, we have reviewed the renal biopsy findings in a large group of patients with T2D, explored pathologies associated with particular renal presentations, and examined their relationship to renal and survival outcomes.

\section{STUDY POPULATION AND METHODS}

Auckland is the largest city in New Zealand (population 1.4 million) and has a high prevalence of obesity and T2D particularly among the indigenous Māori and migrants from island nations in the South Pacific (Pāsifika). ${ }^{10} 11$ More than half of the patients starting renal replacement therapy in Auckland have T2D. ${ }^{12}$ We identified all patients with T2D who had native renal biopsies performed between 2002 and 2008 and confirmed their diagnosis of T2D from examination of clinical records.

The indications for biopsy included acute presentations with persistent renal impairment following AKI or non-acute presentations in which there were atypical clinical features where other kidney disease was suspected. These features included (1) subnephrotic or nephrotic-range proteinuria and a short duration of T2D $(<5$ years) or no diabetic retinopathy; (2) presence of microscopic hematuria; or (3) progressive CKD without diabetic retinopathy or other complications of diabetes.

All renal biopsies were processed in a single Auckland pathology center and reviewed by a single renal histopathologist (LJZ), who was blinded to the identity of the patient and their clinical records or previous pathology reports. Pathology data on some patients have been reported previously. ${ }^{13}$

\section{Histopathological variables}

Archived slides from paraffin-embedded tissues (stained with hematoxylin \& eosin (H\&E), periodic acid-Schiff (PSA), BSA trichrome and Jones methnamine silver) were examined under light microscopy. Immunofluorescence and electron microscopy were applied to biopsies without nodules to examine glomerular basement membrane thickness (GBM) and to classify immune-complex glomerulonephritides.

Non-sclerosed and sclerosed glomeruli were counted to ascertain degree of scarring. Global glomerulosclerosis was defined as $\leq 4$ open capillary loops or $>75 \%$ of the glomerular circumference adherent to the capsule and collapsed. The degree of glomerular capillary patency in all non-sclerosed glomeruli was estimated to the nearest $5 \%$ (assuming patency of zero for sclerosed glomeruli). Cortical interstitial scarring was estimated to the nearest $5 \%{ }^{13}$

The histological definitions of $\mathrm{DN}$ and the other common renal diseases observed in this study are described in (online supplementary table 1). DN was diagnosed and graded according to the Renal Pathology Society classification ${ }^{14}$ with grades 1 and 2 described as early DN, and grades 3 and 4 mesangial nodules described as advanced DN. In biopsies without nodules, $\mathrm{DN}$ was diagnosed if the GBM was $>500 \mathrm{~nm}$ thick.

\section{Clinical variables}

We collected patients' demographic information, prespecified laboratory and clinical variables, and retinopathy statusat the time of biopsy. Baseline renal function was recorded using the mean of the last available three serum creatinine measurements at least 3 months prior to presentation. Glomerular filtration rate (eGFR) was estimated using Chronic Kidney Disease Epidemiology Collaboration (CKD-EPI) method. ${ }^{15}$

We categorized the presentation as non-acute in the setting of stable CKD or normal renal function at the time of biopsy, or acute according to an increase in baseline serum creatinine of $\geq 27 \mu \mathrm{mol} / \mathrm{L}$, or the need for acute dialysis at presentation. In those without any baseline serum creatinine measurements 3 months prior to their renal biopsy, the development of serum creatinine $>106$ $\mu \mathrm{mol} / \mathrm{L}$ was defined as AKI. ${ }^{16}$

Retinal status was recorded from clinical records at last assessment. Retinal status was independently assessed and graded according to a national grading system, and classified as either: none, minimal-to-moderate, or severe diabetic retinopathy. We calculated the rate of eGFR decline as the difference between eGFR measurements at the time of renal biopsy and the last available prior to ESRD or death, divided by the duration of follow-up.

Our main outcomes of interest were ESRD (defined as the need for renal replacement therapy) and allcause mortality. Outcome data were collected from hospital records. We compared freedom from death and from ESRD, censoring for loss to follow-up. Patients were followed from the time of renal biopsy until study endpoints or December 2015.

\section{Statistical methods}

Results are expressed as mean $\pm \mathrm{SD}$ and were compared using Student's t-test or analysis of variance. Median values were expressed with IQRs and compared using the Wilcoxon rank-sum test. Proportions were compared by the $\chi^{2}$ test. We compared the cumulative survival from ESRD and from all-cause mortality, adjusted for age, body mass index (BMI), ethnicity, gender, proteinuria and eGFR at the time of biopsy, using Kaplan-Meier curves 
with log-rank test. Statistical analysis was performed using STATA software (V.13.1).

This study was approved by the New Zealand Health and Disability Ethics Committees (13/NTB/151) and adhered to the principles in the Declaration of Helsinki.

\section{RESULTS}

We identified 263 patients who had renal biopsies. Laboratory results, electronic prescriptions, retinal screening registry data, hospital records and mortality data were available in all patients. Of the 263 patients,
$37 \%$ were of Māori descent, 29\% Pāsifika, 18\% European and $16 \%$ of other ethnicities. The patients were predominantly male and most were substantially overweight. In 238 patients with documented measurements, $33 \%$ had a BMI of $30-35 \mathrm{~kg} / \mathrm{m}^{2}$ and $36 \%$ had a BMI of $>35 \mathrm{~kg} / \mathrm{m}^{2}$.

At the time of biopsy, $43 \%$ of patients were prescribed insulin and $87 \%$ renin-angiotensin system (RAS) inhibitors. Retinal status was known for 245 patients: diabetic retinopathy was absent in 37\%. Prebiopsy demographics and characteristics are shown in table 1.

Table 1 Baseline characteristics at the time of biopsy

\begin{tabular}{|c|c|c|c|c|c|}
\hline & $\begin{array}{l}\text { Data } \\
(\%)\end{array}$ & $\begin{array}{l}\text { Diabetic nephropathy only } \\
(\mathrm{n}=94)\end{array}$ & $\begin{array}{l}\text { Mixed pathologies } \\
(\mathrm{n}=97)\end{array}$ & $\begin{array}{l}\text { Non-diabetic renal } \\
\text { disease only }(n=72)\end{array}$ & $\mathrm{p}$ Value \\
\hline Age & 100 & $58 \pm 10$ & $55 \pm 11$ & $59 \pm 11$ & 0.12 \\
\hline Male (n (\%)) & 100 & $62(66)$ & $64(66)$ & $39(54)$ & 0.18 \\
\hline Ethnicity (n (\%)) & 100 & & & & \\
\hline Māori/Pāsifika & & $65(69)$ & $70(72)$ & $39(55)$ & \\
\hline European & & $14(15)$ & $13(13)$ & $21(29)$ & 0.31 \\
\hline East or South Asian & & $14(15)$ & $13(14)$ & $11(15)$ & \\
\hline Others & & $1(1)$ & $1(1)$ & $1(1)$ & \\
\hline BMI $\left(\mathrm{kg} / \mathrm{m}^{2}\right)$ & 87 & $33 \pm 6$ & $34 \pm 7$ & $36 \pm 12$ & 0.14 \\
\hline Duration of T2D (years) ${ }^{*}$ & 99 & $10(7-18)$ & $11(5-16)$ & $4(2-10) \dagger \ddagger$ & $<0.001$ \\
\hline $\mathrm{HbA1c}(\mathrm{mmol} / \mathrm{mol})$ & 97 & $64 \pm 20$ & $66 \pm 21$ & $56 \pm 13 \dagger \ddagger$ & 0.002 \\
\hline (\%) & & $8.0 \pm 1.8$ & $8.2 \pm 1.9$ & $7.3 \pm 1.2$ & \\
\hline Systolic BP (mm Hg) & 81 & $145 \pm 18$ & $147 \pm 24$ & $142 \pm 20$ & 0.57 \\
\hline Diastolic BP (mm Hg) & 81 & $81 \pm 10$ & $81 \pm 13$ & $81 \pm 13$ & 0.91 \\
\hline Diabetic retinopathy (n (\%)) & 93 & & & & \\
\hline None & & $17(19)$ & $24(26)$ & 50 (78)† ‡ & \\
\hline Minimal-moderate & & $24(27)$ & $26(28)$ & $12(19)$ & $<0.001$ \\
\hline Severe & & $47(54)$ & $43(46)$ & $2(3) \dagger \ddagger$ & \\
\hline $\begin{array}{l}\text { Estimated GFR* }(\mathrm{mL} / \\
\left.\mathrm{min} / 1.73 \mathrm{~m}^{2}\right)\end{array}$ & 100 & $\begin{array}{l}28 \\
(16-43)\end{array}$ & $\begin{array}{l}28 \\
(16-47)\end{array}$ & $\begin{array}{l}41 \dagger \ddagger \\
(25-71)\end{array}$ & $<0.001$ \\
\hline Proteinuria (mg/day) (n (\%)) & 97 & & & & \\
\hline$\leq 300$ & & $10(11)$ & $8(9)$ & $14(21)$ & \\
\hline $301-3500$ & & $32(35)$ & $31(33)$ & $28(41)$ & 0.01 \\
\hline$>3500$ & & $51(54)$ & $54(58)$ & $26(38) \dagger \ddagger$ & \\
\hline Smoking (n (\%)) & 90 & & & & \\
\hline None & & $39(48)$ & $31(34)$ & $27(44)$ & \\
\hline Previous & & $29(35)$ & $36(39)$ & $22(35)$ & 0.68 \\
\hline Current & & $14(17)$ & $25(27)$ & $13(21)$ & \\
\hline \multicolumn{6}{|l|}{ Prescribed drugs (n (\%)) } \\
\hline RAS inhibitors & 99.5 & $80(93)$ & $87(90)$ & $62(86)$ & 0.69 \\
\hline Aspirin & 100 & $66(68)$ & $61(63)$ & $37(51)$ & 0.09 \\
\hline Statin & 99.5 & $64(69)$ & 72 (74) & $53(74)$ & 0.67 \\
\hline
\end{tabular}

${ }^{*}$ Results expressed as median (IQR).

$\dagger p<0.05$ comparing DN and NDRD groups.

$\ddagger \mathrm{p}<0.05$ comparing NDRD and mixed groups.

$\S p<0.05$ comparing $D N$ and mixed groups.

$\mathrm{BMI}$, body mass index; BP, blood pressure; DN, diabetic nephropathy; GFR, glomerular filtration rate; NDRD, non-diabetic renal disease. 
Ninety-four patients (36\%) had DN alone, $97(37 \%)$ had both DN and NDRD (mixed) and 72 (27\%) had NDRD alone. Patients with NDRD alone differed in having a shorter duration of diabetes, less retinopathy and higher eGFR at time of renal biopsy compared with $\mathrm{DN}$ alone and mixed groups $(\mathrm{p}<0.001)$. The absence of diabetic retinopathy (OR 20.2, 95\% CI 7.2 to 56.1, $\mathrm{p}<0.001)$ was strongly associated with NDRD.

\section{Non-diabetic renal disease}

Among patients with NDRD and mixed disease, focal segmental glomerulosclerosis (FSGS) was the most common non-diabetic pathology found in 78 patients
(46\%): $24(31 \%)$ as sole NDRD, $32(41 \%)$ together with DN and $22(28 \%)$ in combination with other NDRD pathologies. Patients with any FSGS (alone or mixed) had higher BMI than those with other renal pathologies (NDRD, DN or mixed) $\left(35.8 \pm 8.1\right.$ vs $33.1 \pm 7.1 \mathrm{~kg} / \mathrm{m}^{2}$, $\mathrm{p}=0.012$ ).

Other common NDRD pathologies (mixed and alone) included interstitial nephritis $(\mathrm{n}=40,24 \%)$, postinfectious glomerulonephritis $(\mathrm{n}=14,8 \%)$, membranous glomerulonephritis $(\mathrm{n}=8,5 \%)$ and minimal change disease $(n=6,3.5 \%)$. Interstitial nephritis was commonly observed in combination with DN: in total, 34 patients

Table 2 Characteristics of patients with acute and non-acute presentations

\begin{tabular}{|c|c|c|c|}
\hline & Acute & Non-acute & p Value \\
\hline Number (\%) & $146(56)$ & $116(44)$ & - \\
\hline Age (years) & $57 \pm 11$ & $57 \pm 11$ & 0.58 \\
\hline Male (\%) & $94(64)$ & $70(61)$ & 0.50 \\
\hline $\mathrm{BMI}\left(\mathrm{kg} / \mathrm{m}^{2}\right)$ & $34 \pm 14$ & $34 \pm 13$ & 0.37 \\
\hline Duration of diabetes (years) ${ }^{\star}$ & $10(5-17)$ & $8(4-13)$ & 0.05 \\
\hline Retinopathy grade (n (\%)) & $\mathrm{n}=131$ & $n=113$ & \\
\hline None & $30(23)$ & $61(54)$ & \\
\hline Minimal-moderate & $35(27)$ & $27(24)$ & $<0.001$ \\
\hline Severe & $66(50)$ & $25(22)$ & \\
\hline Proteinuria $\left(\mathrm{g} /\right.$ day) ${ }^{*}$ & $5.0(1.4-10.5)$ & $2.9(1.0-5.3)$ & 0.001 \\
\hline \multicolumn{4}{|l|}{ Estimated GFR $\left(\mathrm{mL} / \mathrm{min} / 1.73 \mathrm{~m}^{2}\right)^{\star}$} \\
\hline At biopsy & $21(12-32)$ & $47(35-71)$ & $<0.001$ \\
\hline 3 months before biopsy & $43(30-58)$ & $48(34-72)$ & 0.07 \\
\hline \multicolumn{4}{|l|}{ Histological diabetic nephropathy (n (\%)) } \\
\hline Absent & $25(17)$ & $47(41)$ & \\
\hline Grades 1 and 2 & $15(10)$ & $19(16)$ & $<0.001$ \\
\hline Grades 3 and 4 & $106(73)$ & $50(43)$ & \\
\hline \multicolumn{4}{|l|}{ Non-diabetic renal diseases (n (\%)) } \\
\hline Focal segmental glomerulosclerosis & $18(12.5)$ & $38(33)$ & $<0.001$ \\
\hline Membranous nephropathy & $2(1.5)$ & $6(5)$ & 0.08 \\
\hline Minimal change disease & $0(0)$ & $6(5)$ & 0.005 \\
\hline IgA nephropathy & $0(0)$ & $3(2.5)$ & 0.05 \\
\hline Interstitial nephritis & $32(22)$ & $8(7)$ & 0.001 \\
\hline Immune complex glomerulonephritides & $21(14.5)$ & $4(3.5)$ & 0.003 \\
\hline Acute tubular necrosis & $9(6)$ & $0(0)$ & 0.007 \\
\hline Acute pyelonephritis & $3(2)$ & $1(1)$ & 0.43 \\
\hline Others & $2(1.5)$ & $7(6)$ & 0.04 \\
\hline \multicolumn{4}{|l|}{ Histological findings } \\
\hline DN grade* & $3(2-4)$ & $2(0-3)$ & $<0.001$ \\
\hline Sclerosed glomeruli (\%) & $35(17-54)$ & $30(11-51)$ & 0.24 \\
\hline Glomerular capillary patency (\%) & $50(25-73)$ & $80(40-95)$ & $<0.001$ \\
\hline Interstitial scarring (\%) & $25(15-49)$ & $20(10-35)$ & $<0.001$ \\
\hline
\end{tabular}

${ }^{*}$ Results expressed as median (IQR).

BMI, body mass index; DN, diabetic nephropathy; GFR, glomerular filtration rate. 
$(35 \%)$ in the mixed group had histological evidence of interstitial nephritis.

The NDRD group had a greater median glomerular capillary patency, fewer sclerosed glomeruli and less interstitial scarring than the DN group $(p<0.001)$. The mixed group had intermediate values (online supplementary table 2 ).

\section{Acute versus non-acute presentations}

Two hundred and sixty-two patients had a serum creatinine measurement at the time of the renal biopsy. Of these, 146 patients $(56 \%)$ had a renal biopsy for the indication of AKI. In those with acute presentations, the median (IQR) serum creatinine at the time of biopsy was $253(186-400) \mu \mathrm{mol} / \mathrm{L}$, and the baseline creatinine (3 months earlier) had been 143 (100-176) $\mu \mathrm{mol} / \mathrm{L}$. Twenty-four patients with no prior documented serum creatinine measurements had a serum creatinine of $>106$ $\mu \mathrm{mol} / \mathrm{L}$ at the time of renal biopsy in keeping with AKI, this included six patients with severe AKI. Among these 24 patients, the median (IQR) serum creatinine at the time of biopsy was 213 (175-351) $\mu \mathrm{mol} / \mathrm{L}$.

In those with acute presentations, $40 \%$ had pure $\mathrm{DN}$. Interstitial nephritis and immune complex glomerulonephritides were the most common NDRD pathologies occurring in addition to $\mathrm{DN}$ or as sole pathologies. DN (mixed and alone) was observed more commonly in patients who presented with AKI than in those with non-acute presentations ( $83 \%$ vs $59 \%, \mathrm{p}<0.001)$. Patients with acute presentations had a higher prevalence of advanced DN lesions (grades 3-4). Acute presentations were associated with more severe $\mathrm{DN}$, a higher percentage of interstitial scarring and reduce capillary patency reported on histopathology.

Of the 116 patients who presented non-acutely, the serum creatinine at the time of biopsy was 121 (93-170) $\mu \mathrm{mol} / \mathrm{L} .9 .5 \%$ had CKD stage 1, 26\% CKD stage 2, 20.5\% CKD stage $3 \mathrm{a}, 26 \%$ CKD stage 3b, 14.5\% CKD stage $4 \%$ and $3.5 \%$ CKD stage $5 .{ }^{17}$ FSGS (mixed and alone) was the most common NDRD pathology in this group (table 2).

Renal biopsy findings resulted in a change of treatment in 31 (12\%) patients: immunosuppressive and steroid therapies were prescribed in 21 and 7 patients in the NDRD and the mixed groups, respectively; in addition, one patient received mephalan for amyloidosis, one received lamivudine for hepatitis B-associated membranoproliferative glomerulonephritis, and statin treatment was stopped in one patient with biopsy-proven immune-complex glomerulonephritis. Eleven $(35 \%)$ of the 31 patients who received a change in their treatment following their renal biopsy subsequently needed renal replacement therapy compared with $72(52 \%)$ patients whose management was unchanged. Adjusted for age, ethnicity, gender, BMI, degree of proteinuria and eGFR at presentation, renal prognosis in the former group was better, although not statistically significant $(\mathrm{p}=0.058)$.

\section{Duration of diabetes}

Patients were further analysed according to whether any DN or any NDRD was present in their biopsy histology; thus, in this analysis, patients with mixed pathologies were represented in both groups. The proportion of

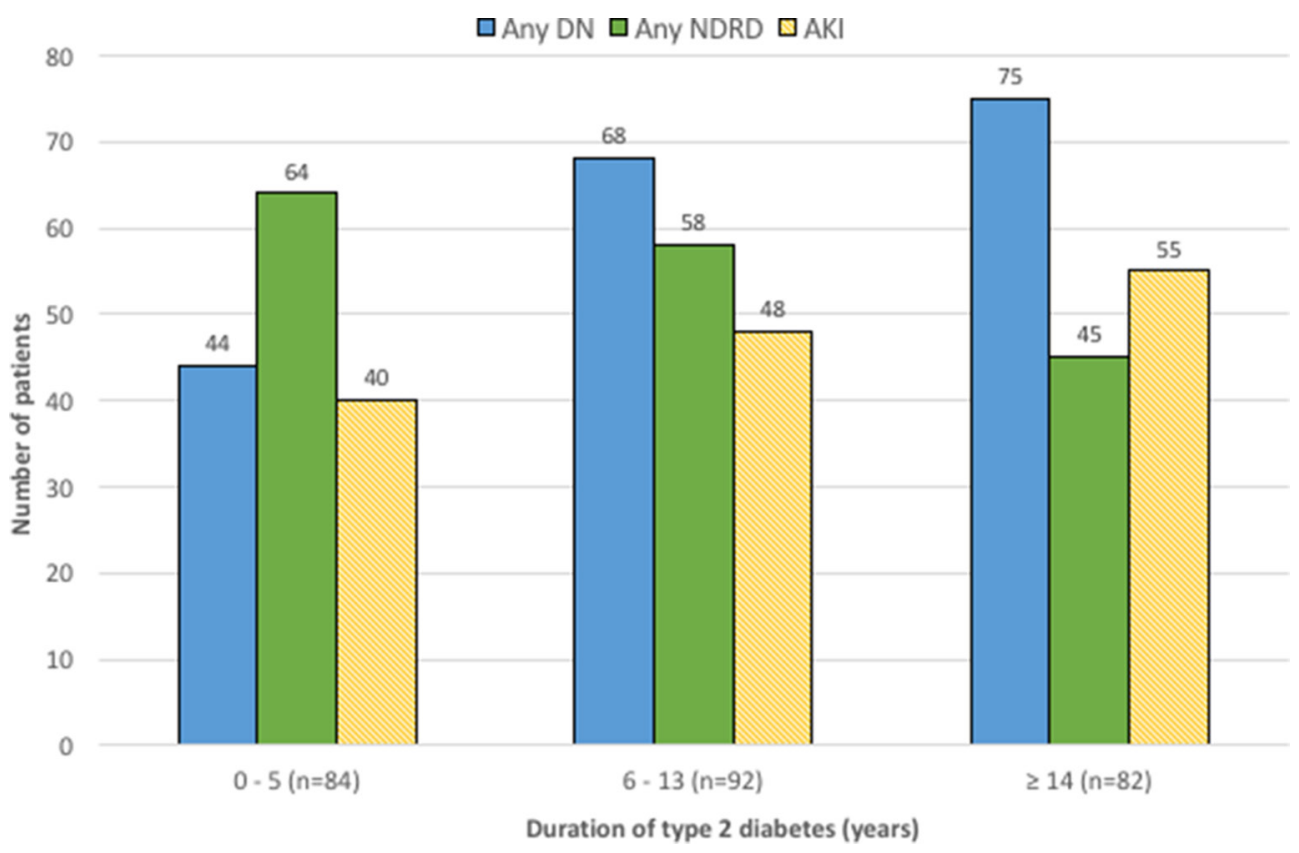

Figure 1 Relationship between time from diagnosis of type 2 diabetes to renal biopsy and the proportion of biopsies with any diabetic nephropathy (DN) and any non-diabetic renal disease (NDRD) $(n=258)$. The proportion of any DN increased with duration of type 2 diabetes $(p<0.005)$, but NDRD remained a common finding even in patients with a long duration of type 2 diabetes. The proportion of acute presentations was similar in all three tertiles of diabetes duration $(p=0.18)$. AKI, acute kidney injury. 


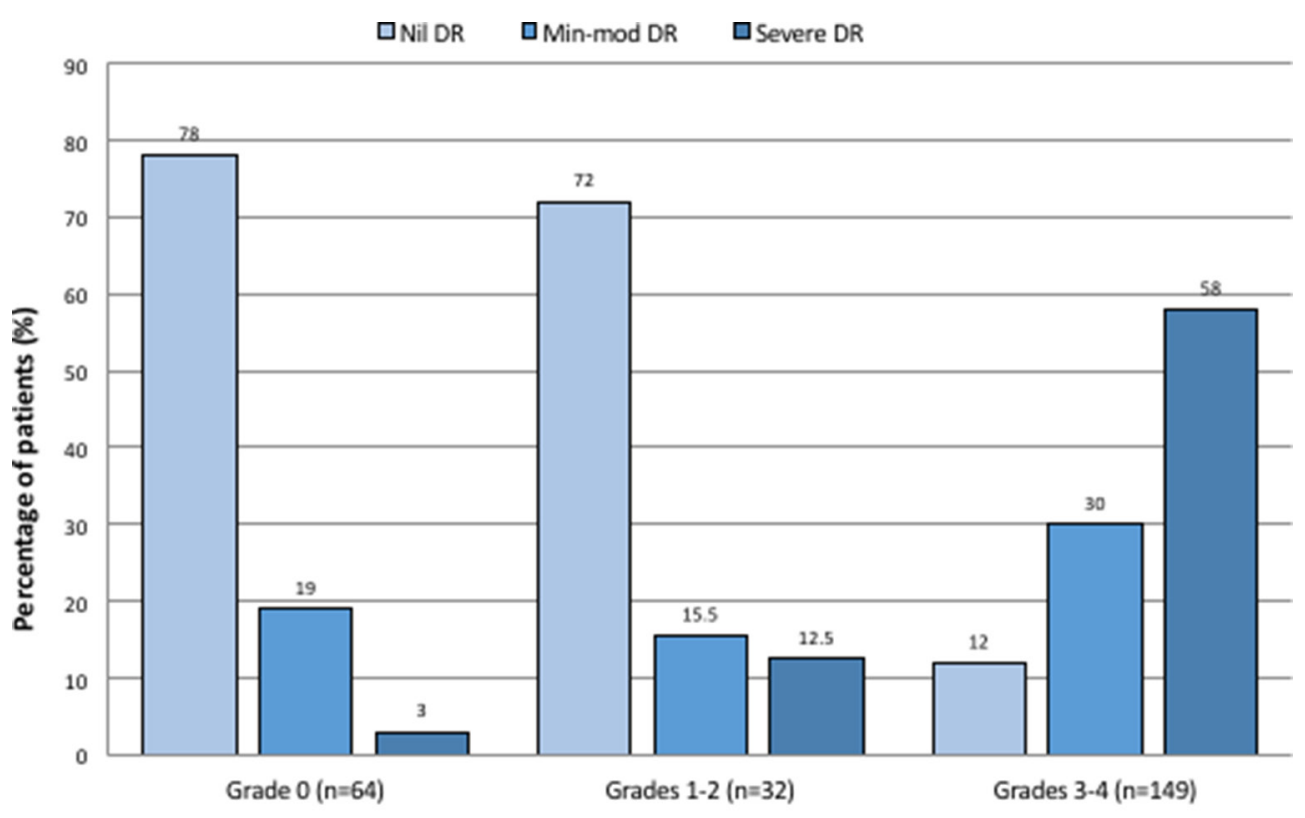

DN histopathological grade

Figure 2 Relationship between grades of diabetic retinopathy and severity of diabetic renal lesions $(n=245)$. More severe diabetic renal lesions were strongly related to the severity of diabetic retinopathy in patients with diabetic nephropathy (DN) $(p<0.001)$.

biopsies showing any $\mathrm{DN}$ increased with diabetes duration: $52 \%$ in those with $\leq 5$ years, $74 \%$ with $6-13$ years and $92 \%$ with $\geq 14$ years duration $(p<0.001)$. Although the proportion with any NDRD was lower in those with diabetes of long duration, NDRD was still found in $55 \%$ of patients with $\geq 14$ years of T2D (figure 1). Episodes of AKI were was high and similar in all tertiles $(\mathrm{p}=0.18)$.

In patients without diabetic retinopathy, most $(80 \%)$ had either no or early (grades 1 and 2) diabetic renal lesions, but in those with more severe retinal disease, the majority (93.5\%) had advanced diabetic renal lesions (grades 3 and 4). The degree of DN lesions correlated strongly with the severity of retinopathy $(p<0.001$, figure 2).

\section{Renal and survival outcomes}

After follow-up of 0.1-13.6 (median 3.6) years, 133 renal events and 61 deaths occurred.

Seventeen $(24 \%)$ patients in the NDRD group reached ESRD-a lower proportion than the Mixed (68\%) and DN $(53 \%)$ groups $(\mathrm{p}<0.001$, figure $3 \mathrm{a})$. The median (IQR) rate of eGFR decline in the NDRD group was significantly
A

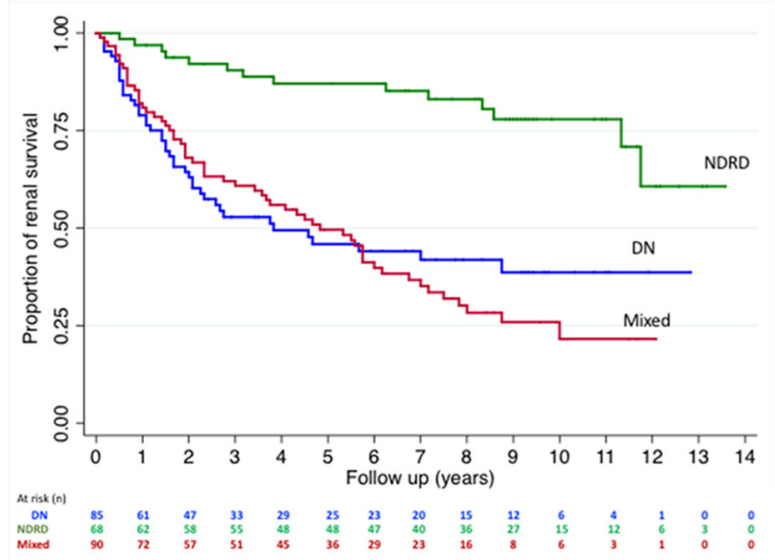

B

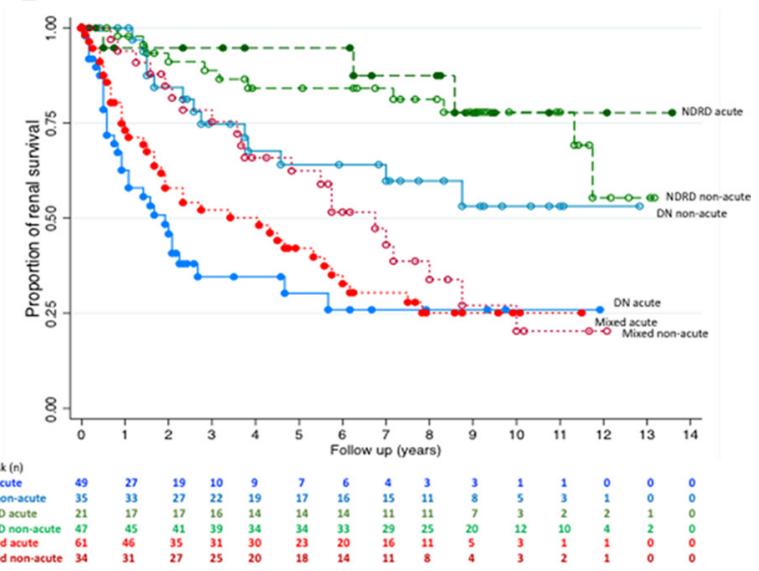

Figure 3 (A) Kaplan-Meier renal survival estimates of patients with diabetic nephropathy (DN), non-diabetic renal disease (NDRD) and mixed groups, adjusting for age, ethnicity, BMI, gender, proteinuria and eGFR at the time of biopsy. Patients with NDRD had significantly better renal prognosis (Log-rank, $p<0.001$ ). (B) Kaplan-Meier renal survival estimates of patients with DN, all types of NDRD and mixed renal pathologies (Mixed) presenting acutely (solid circles) and non-acutely (hollow circles), adjusted for age, ethnicity, BMI, gender, proteinuria and eGFR at the time of biopsy. Patients with DN presenting acutely had the poorest renal prognosis $(p<0.001)$. BMI, body mass index; eGFR, estimated glomerular filtration rate. 


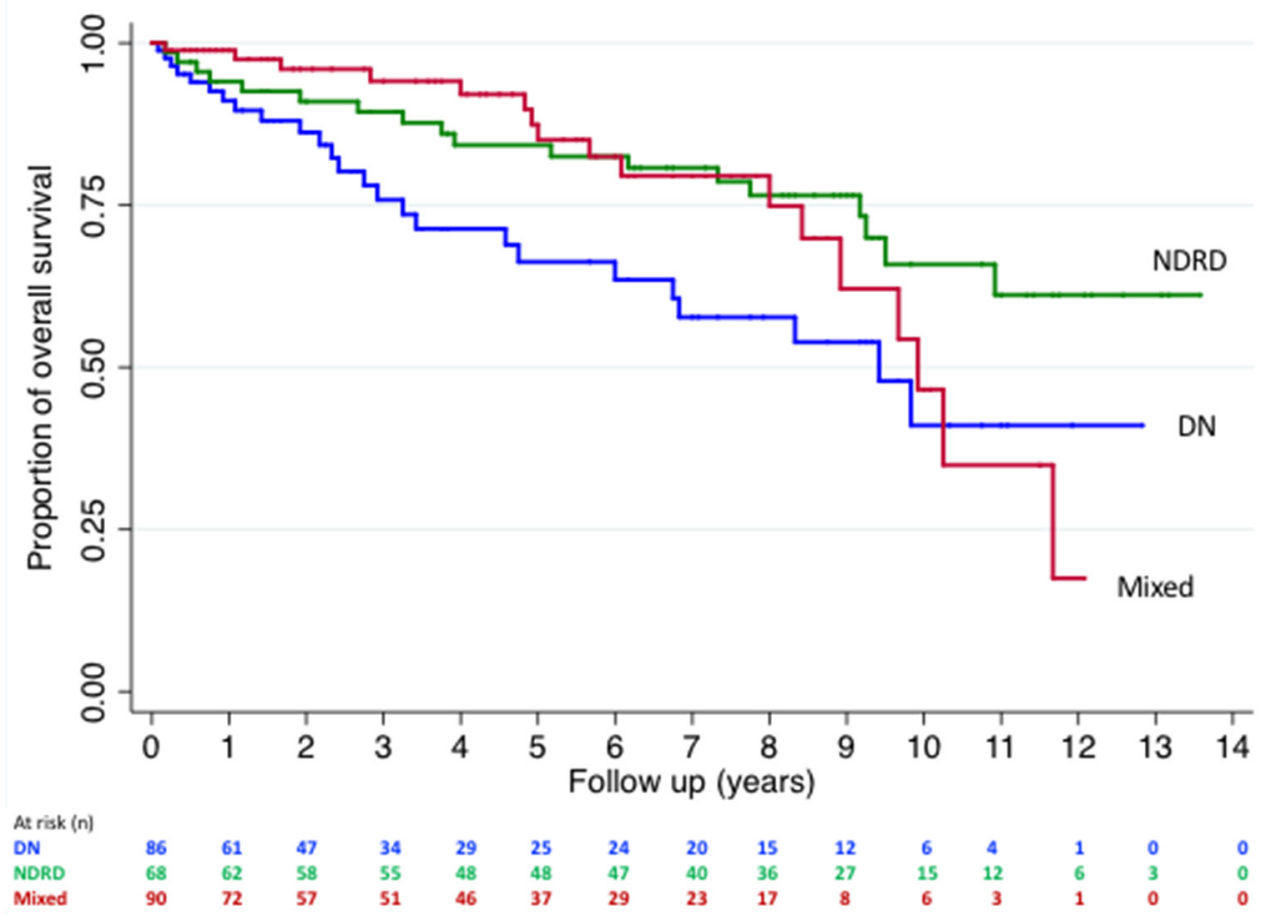

Figure 4 Kaplan-Meier survival estimates of patients with diabetic nephropathy (DN), non-diabetic renal disease (NDRD) and mixed groups adjusting for age, ethnicity, BMI, gender, proteinuria and eGFR at the time of biopsy. Overall survival from allcause mortality was worse in the DN group (Log-rank, $p=0.025)$. BMI, body mass index; eGFR, estimated glomerular filtration rate.

slower at $2.2(-0.1-5.8) \mathrm{mL} / \mathrm{min} / 1.73 \mathrm{~m}^{2} /$ year than the DN and mixed groups $(8.3(3.0-17.2)$ and 6.9 (2.0-12.4) $\mathrm{mL} / \mathrm{min} / 1.73 \mathrm{~m}^{2} /$ year, respectively, $\left.\mathrm{p}<0.001\right)$.

Patients with early DN lesions had a better renal prognosis than those with advanced lesions (Log-rank, $\mathrm{p}<0.001)$. Renal survival was significantly worse in patients who presented with AKI (Log-rank, $p<0.001$ ), especially in those with $\mathrm{DN}$ when compared with all types of NDRD (figure 3b). Renal prognosis was similar in the NDRD group in those presenting acutely or non-acutely (Log-rank, $\mathrm{p}=0.61$ ). Patients with $\mathrm{DN}$ were 4.5 times more likely to reach ESRD than those with other non-diabetic CKD $(p=0.002)$. Patients with mixed pathologies had an intermediate renal prognosis.

Over the median 3.6-year follow-up, the overall survival was poorest in the DN group compared with the mixed and NDRD groups (Log-rank, $\mathrm{p}=0.025$ ) (figure 4).

\section{DISCUSSION}

Our study confirms that in patients with renal disease and T2D who are selected for renal biopsy, there is marked pathological heterogeneity. More than half of our patients had another form of kidney disease, which is a higher proportion than reported in a recent meta-analysis of 48 studies. ${ }^{3}$ We note that this meta-analysis was not exactly comparable, in that it included studies of patients with type 1 as well as type 2 diabetes. It also included studies in which biopsies were performed for research purposes as opposed to clinical indications. ${ }^{18}$ IgA nephropathy was the most prevalent NDRD reported in the meta-analysis and probably reflects of the high percentage of studies in Asian populations. In contrast, FSGS was the most prevalent NDRD in our study, consistent with observations in studies performed among other predominantly European populations. ${ }^{16}{ }^{19-21}$ Our results are arguably a more realistic reflection of clinical presentations of renal disease with T2D in developed nations.

In keeping with previous reports, we also found that the renal prognosis is generally better with NDRD. ${ }^{6-9}$ Our analysis differs in that it strongly links this difference in renal prognosis to the mode of presentation. It also reinforces the value of renal biopsy in patients with T2D. In our study, renal biopsies led to a change of treatment in $12 \%$ of our cohort, with the renal prognosis appearing to be improved.

As expected, the proportion of cases with biopsy-proven DN increased with diabetes duration, and the severity of retinopathy also correlated strongly with the severity of renal diabetic lesions. Patients with DN had greater degrees of glomerular sclerosis, interstitial fibrosis and poorer capillary patency compared with those with NDRD. The severity of the diabetic lesions was closely linked to renal survival.

We found that DN was more commonly present in patients presenting with AKI than in those with non-acute presentations, and they also had more advanced diabetic renal lesions. Most patients with DN presenting with AKI had superimposed interstitial nephritis, immune-complex 
glomerulonephritis or acute tubular necrosis, but in a substantial proportion, no additional pathology was identified. An acute presentation was associated with poorer renal prognosis, especially in patients with underlying DN. These findings are in keeping with recent observations that patients with diabetes are particularly susceptible to $\mathrm{AKI}^{4}$ and that episodes of AKI hasten the onset of ESRD ${ }^{5}$ with the infiltration of inflammatory cells and/or progressive loss of interstitial capillaries contributing to worsening renal scarring and ischemic injury. ${ }^{22} 23$

In patients with NDRD alone, the mode of presentation did not significantly impact renal prognosis. Patients in the NDRD and mixed group displayed a lesser degree of glomerular sclerosis, interstitial scarring and greater capillary patency on histology, which likely reflect an underlying greater renal reserve. Furthermore alterations in medical management after biopsy may have changed the natural progression of the renal disease in some of those presenting acutely.

In non-acute presentations (typically with heavy proteinuria, a short duration of T2D and little or no retinopathy), FSGS was the dominant pathology in both the NDRD and mixed groups. The prevalence of FSGS in our population was greater than that seen in studies from other countries. ${ }^{3}$ The incidence of obesity-related FSGS has steadily increased over time ${ }^{24}$ and carries a substantial risk of progressive renal decline. ${ }^{25}$ High rates of proteinuric renal disease (independent of diabetes) have previously been observed in Māori and Pāsifika people, ${ }^{26}$ and this likely reflects a high incidence of obesity-related glomerulopathy and associated FSGS. ${ }^{13}$ Patients with FSGS in our study were notably more overweight than those with other renal pathologies. A high BMI is also independently associated with the progression of CKD. Proposed mechanisms include maladaptive changes and glomerular hypertension resulting in glomerulosclerosis and podocyte strain. ${ }^{27} 28$

A faster decline of eGFR has been demonstrated in proteinuric patients in those with severe retinopathy ${ }^{729}$; however, renal prognosis may improve if remission of proteinuria can be achieved..$^{29-32}$ Management of diabetic renal disease has largely been focused on remission of proteinuria through blood pressure lowering and the antiproteinuric effects of ACE inhibitors and angiotensin II receptor blockers. ${ }^{25}$ While RAS blockade remains the mainstay treatment in renal disease in people with diabetes, a large number of patients with T2D continue to progress to ESRD. Trials of novel treatments have had little success. ${ }^{3033}$ These studies however were conducted without histopathological diagnoses, and it is perhaps unrealistic to expect a single treatment modality to be effective in such a heterogeneous disorder.

In the other studies that have shown a better renal prognosis for NDRD, the predominant pathologies $(55 \%-71 \%)$ were mostly associated with a slowly progressive disease course: IgA nephropathy, membranous nephropathy, FSGS or minimal change disease. ${ }^{6-9}$ Thus, NDRD typically associated with a CKD presentation (predominantly FSGS in our population) has a more benign natural history than DN. However, overall survival was less affected by the etiology of renal disease or the severity of diabetic lesions, probably because of high cardiovascular mortality. ${ }^{34}$

There are several limitations to this study. First, $78 \%$ of the renal biopsies were from Māori and Pāsifika patients, so our findings may not be entirely generalizable to other populations. However, $69 \%$ of the patients in this cohort are obese, and it may be argued that there are more similarities than differences to populations with T2D and renal disease in other developed countries. Second, there is an inevitable sampling error associated with renal biopsy. Thirdly, selection bias is unavoidable because only patients with atypical presentations were biopsied. As such, it could be argued that the prevalence and significance of NDRD is exaggerated by studies such as ours. There is, however, reason to believe that in our population at least, NDRD may be much more common than is generally appreciated. In patients with T2D and renal disease, the absence of diabetic retinopathy is strongly suggestive of NDRD, ${ }^{35}$ and in our population a third of T2D patients with proteinuric renal disease have no retinopathy when macroalbuminuria is first detected. ${ }^{30}{ }^{36}$ Although knowledge of renal histopathology led to a change in management of 1 in 8 patients (most commonly the introduction of immunosuppressive therapy), the small numbers involved meant we could not determine whether this change was associated with improved outcomes. This is an important research priority, but would require a large number of patients for sufficiently powered analyses. Finally, morphological features of FSGS and their etiological associations are yet to be formally established, making the distinction between primary and secondary FSGS difficult and open to question. ${ }^{37}$

\section{Conclusion}

Our study supports the view that a diverse range of pathologies additional to DN can contribute to CKD in patients with T2D and that the underlying histological diagnosis and mode of presentation have important prognostic implications to renal survival.

The prevalence and severity of DN increases with longer diabetes duration and with more severe glomerular lesions comes greater loss of renal function. At any stage acute insults can supervene. Patients with DN are particularly vulnerable to AKI, which can cause permanent loss of renal function and worsen renal prognosis.

The data suggest a dynamic model of renal disease in T2D in which DN can have complex interactions with other pathologies. A more liberal renal biopsy policy might permit better recognition of NDRD and more personalized approaches to treatment, which may impact patient outcomes and the burden of ESRD. CKD progression from obesity-related FSGS might be an appropriate target for intervention, and further study of interventions such as bariatric surgery should be a priority. ${ }^{28} 38$ 
Contributors JT and TC were involved in the research idea and study design. JT and LJZ were involved in data acquisition and along with TC were involved in data analysis, interpretation and reporting of study results. JT conducted the statistical analysis. LJZ, JFC, MRM and JT were involved in the manuscript editing and final approval.

Funding This work has been supported by the New Zealand Diabetes Foundation Fellowship, which had been awarded to JT between June 2013 and June 2014.

Competing interests JZ, LJZ, JFC and TC have nothing to declare. MRM is a full-time employee of Baxter Healthcare (Asia-Pacific) Ltd, a part-time employee of University of Auckland as an adjunct associate professor, and a part-time employee of Counties Manukau Health (New Zealand) as a clinical nephrologist.

Patient consent This is a retrospective analysis, and local ethics approval has been obtained prior to the start of this study.

Ethics approval Auckland, New Zealand.

Provenance and peer review Not commissioned; externally peer reviewed.

Data sharing statement This was a retrospective study, and all data have been presented in this manuscript. All authors agreed to provide additional information for further analysis.

Open Access This is an Open Access article distributed in accordance with the Creative Commons Attribution Non Commercial (CC BY-NC 4.0) license, which permits others to distribute, remix, adapt, build upon this work non-commercially, and license their derivative works on different terms, provided the original work is properly cited and the use is non-commercial. See: http://creativecommons.org/ licenses/by-nc/4.0/

(C) Article author(s) (or their employer(s) unless otherwise stated in the text of the article) 2017. All rights reserved. No commercial use is permitted unless otherwise expressly granted.

\section{REFERENCES}

1. Plantinga LC, Crews DC, Coresh J, et al. Prevalence of chronic kidney disease in US adults with undiagnosed diabetes or prediabetes. Clin J Am Soc Nephrol 2010;5:673-82.

2. Macisaac RJ, Jerums G. Diabetic kidney disease with and without albuminuria. Curr Opin Nephrol Hypertens 2011;20:246-57.

3. Fiorentino M, Bolignano D, Tesar V, et al. Renal biopsy in patients with diabetes: a pooled meta-analysis of 48 studies. Nephrol Dial Transplant 2017;32:97-110.

4. James MT, Grams ME, Woodward M, et al. A Meta-analysis of the Association of estimated GFR, Albuminuria, Diabetes Mellitus, and hypertension with acute kidney Injury. Am J Kidney Dis 2015;66:602-12.

5. Coca SG, Singanamala S, Parikh CR, et al. Chronic kidney disease after acute kidney injury: a systematic review and meta-analysis. Kidney Int 2012;81:442-8.

6. Chang TI, Park JT, Kim JK, et al. Renal outcomes in patients with type 2 diabetes with or without coexisting non-diabetic renal disease. Diabetes Res Clin Pract 2011;92:198-204.

7. Oh SW, Kim S, Na KY, et al. Clinical implications of pathologic diagnosis and classification for diabetic nephropathy. Diabetes Res Clin Pract 2012;97:418-24.

8. Soleymanian T, Hamid G, Arefi M, et al. Non-diabetic renal disease with or without diabetic nephropathy in type 2 diabetes: clinical predictors and outcome. Ren Fail 2015;37:572-5.

9. Wong TY, Choi PC, Szeto CC, et al. Renal outcome in type 2 diabetic patients with or without coexisting nondiabetic nephropathies. Diabetes Care 2002;25:900-5.

10. Statistics New Zealand. Census QuickStats about Māori, 2013.

11. Statistics New Zealand and Ministry of Pacific Island Affairs. Demographics of New Zealand's Pacific population, 2010.

12. Hughes J, Palmer S. End stage kidney disease among indigenous peoples of Australia and New Zealand. Australia New Zealand dialysis and transplant (ANZDATA) Registry. 37th annual Repor. Adelaide, South Australia, 2014.
13. Zwi LJ, Yiu TS, Marshall MR, et al. Non-diabetic renal diseases in a multi-ethnic New Zealand cohort with type 2 diabetes mellitus: clinical and histopathological features. Pathology 2014;46:424-32.

14. Tervaert TW, Mooyaart AL, Amann K, et al. Renal Pathology Society. pathologic classification of diabetic nephropathy. J Am Soc Nephrol 2010;21:556-63.

15. Levey AS, Stevens LA, Schmid CH, et al. A new equation to estimate glomerular filtration rate. Ann Intern Med 2009;150:604-12.

16. Sharma SG, Bomback AS, Radhakrishnan J, et al. The modern spectrum of renal biopsy findings in patients with diabetes. Clin $\mathrm{J}$ Am Soc Nephrol 2013;8:1718-24.

17. Kidney disease: improving global outcomes (KDIGO) CKD Work Group. KDIGO 2012 clinical Practice Guideline for the evaluation and management of chronic kidney disease. Kidney Int Supp/ 2013;3:1-150.

18. Caramori ML. Should all patients with diabetes have a kidney biopsy? Nephrol Dial Transplant 2017;32:3-5.

19. Pham TT, Sim JJ, Kujubu DA, et al. Prevalence of nondiabetic renal disease in diabetic patients. Am J Nephrol 2007;27:322-8.

20. Horvatic I, Tisljar M, Kacinari P, et al. Non-diabetic renal disease in croatian patients with type 2 diabetes mellitus. Diabetes Res Clin Pract 2014;104:443-50.

21. Pallayova M, Mohammed A, Langman G, et al. Predicting nondiabetic renal disease in type 2 diabetic adults: the value of glycated hemoglobin. J Diabetes Complications 2015;29:718-23.

22. Sean Eardley K, Cockwell P. Macrophages and progressive tubulointerstitial disease. Kidney Int 2005;68:437-55.

23. Fine LG, Bandyopadhay D, Norman JT. Is there a common mechanism for the progression of different types of renal diseases other than proteinuria? towards the unifying theme of chronic hypoxia. Kidney Int Suppl 2000;75:S22-6.

24. Kambham N, Markowitz GS, Valeri AM, et al. Obesityrelated glomerulopathy: an emerging epidemic. Kidney Int 2001;59:1498-509.

25. Stanton RC. Clinical challenges in diagnosis and management of diabetic kidney disease. Am J Kidney Dis 2014;63:S3-S21.

26. Thompson CF, Simmons D, Collins JF, et al. Predisposition to nephropathy in Polynesians is associated with family history of renal disease, not diabetes mellitus. Diabet Med 2001;18:40-6.

27. de Vries AP, Ruggenenti P, Ruan XZ, et al.Fatty kidney:emerging role of ectopic lipid in obesity-related renal disease. Lancet Diabetes Endocrinol 2014;2:417-26.

28. Wickman C, Kramer H. Obesity and kidney disease: potential mechanisms. Semin Nephrol 2013;33:14-22.

29. Trevisan R, Vedovato M, Mazzon C, et al. Concomitance of diabetic retinopathy and proteinuria accelerates the rate of decline of kidney function in type 2 diabetic patients. Diabetes Care 2002;25:2026-31.

30. Tan J, Jaung R, Gamble G, et al. Proteinuric renal disease in type 2 diabetes-is remission of proteinuria associated with improved mortality and morbidity? Diabetes Res Clin Pract 2014;103:63-70.

31. Rossing K, Christensen PK, Hovind P, et al. Remission of nephroticrange albuminuria reduces risk of end-stage renal disease and improves survival in type 2 diabetic patients. Diabetologia 2005;48:2241-7.

32. Retnakaran R, Cull CA, Thorne KI, et al. Risk factors for renal dysfunction in type 2 diabetes: U.K. prospective Diabetes Study 74. Diabetes 2006;55:1832-9.

33. Khan SS, Quaggin SE. Therapies on the Horizon for Diabetic Kidney Disease. Curr Diab Rep 2015;15:1-8.

34. Barkoudah $\mathrm{E}$, Skali $\mathrm{H}$, Uno $\mathrm{H}$, et al. Mortality rates in trials of subjects with type 2 diabetes. J Am Heart Assoc 2012;1:8-15.

35. He F, Xia X, Wu XF, et al. Diabetic retinopathy in predicting diabetic nephropathy in patients with type 2 diabetes and renal disease: a meta-analysis. Diabetologia 2013;56:457-66.

36. Tan J, McCready F, Noovao F, et al. Intensification of blood pressure treatment in Pasifika people with type 2 diabetes and renal disease: a cohort study in primary care. N Z Med J 2014;127:17-26.

37. D'Agati VD, Fogo AB, Bruijn JA, et al. Pathologic classification of focal segmental glomerulosclerosis: a working proposal. Am J Kidney Dis 2004;43:368-82.

38. Miras AD, Chuah LL, Khalil N, et al. Type 2 diabetes mellitus and microvascular complications 1 year after Roux-en-Y gastric bypass: a case-control study. Diabetologia 2015;58:1443-7. 